\title{
Proceedings of the meeting of the Society of British Neurological Surgeons, Salford, 13-14 April 1989
}

\section{EARLY ANEURYSM SURGERY - A REVIEW RAC Jones. Salford}

In an era of increasing enthusiasm for early operation for ruptured aneurysm, the author explored the factors which delayed referral to neurosurgery and reviewed the results of an aggressive management policy in a personal consecutive series of 94 patients with saneurysmal rupture over a five year period. Despite the promotion of CT as the best immediate diagnostic device, admission of $70 \%$ of patients to their District Hospital on the day of admission and referral of $70 \%$ of these patients to the Neurosurgical - Unit within a day of their initial admission, nevertheless $37 \%$ of the patients were subjected to lumbar puncture as the first diagnostic procedure and $30 \%$ suffered a delay of four days or more from onset of symptoms to neurosurgical admission.

In a review of those 32 patients who had 'early' operation, within three days of haemorrhage, there was an overall mortality for all grades and aneurysm sites, of $22 \%$ (seven patients); 19 patients $(60 \%)$ achieved a favourable outcome.

THE MAUDSLEY MENTATION TEST: A METHOD FOR MEASUREMENT OF FLUCTUATIONS IN COGNITIVE FUNCTION IN PATIENTS UNDERGOING SURGERY FOR INTRACRANIAL ANEURYSM

AW Darkins, AJ Strong, $M$ von Schoenberg, M Wyke, I Daum. Maudsley Hospital, London

Fluctuations in alertness occur following aneurysmal subarachnoid haemorrhage (SAH), but existing scales do not provide sufficient resolution for detailed measurement of their frequency and extent. Also, the impact of late neuropsychological residua upon a patient's ability to resume his pre-morbid lifestyle is increasingly recognised; a method is required which will allow comparison of fluctuations in psychological function during the peri-operative phase with late, fixed neuropsychological deficits.

The Maudsley Mentation Test has been devised with the aim of providing a bedside measurement scale for use primarily by nurs- ing staff to evaluate neuropsychological function at regular intervals ( 2 hourly) during the perioperative period. A twelve-point scale based on simple tasks was devised, with emphasis on the requirements that (1) the time taken to administer the tests should be brief-no more than five minutes-and (2), several equivalent versions could be constructed so that the patient could be assessed every few hours with a new version, so as to avoid practice effects. Each version comprised five different types of cognitive "probe", with two questions per probe, making a total of 10 tasks. Six versions were devised and assessed initially. The first probe tests the subject's ability to carry out two stage commands. For instance, the patient is asked to raise a hand and then touch his ear. The second probe requires the subjects to complete a reversed sequencing task. Further probes assess verbal function and simple calculation ability.

An inter-rater reliability study was conducted by two neurosurgeons, two nurses and two psychologists in 27 neuropsychiatric patients with stable deficits. Data were subjected to classical item analysis using the software package PSPX. Reliability coefficient (Cronbach's alpha) was 0.890.91: item-scale correlations were significant at the $1 \%$ level. Results were consistent, and were independent of the status of the examiner and the particular version used.

Initial experience with patients with aneurysmal subarachnoid haemorrhage confirms that since the probes address a wide range of areas of cognitive function, the final score is non-specific and may be affected by deficits in particular areas such as speech or frontal lobe function. A low score may for example reflect lack of cooperation: we have also seen instances in which a patient may obtain a full score but be disorientated. However, wide fluctuations in total score are also seen, and it may therefore prove possible to use the test in order to provide greater resolution, both in time and in severity of deficit, than is available from a 2-point test such as orientation. Ability to quantify such fluctuations is an essential prerequisite for the evaluation of treatment regimens intended or believed to produce rapid improvements in function.
BRAINSTEM VASCULAR MALFORMATIONS: DIAGNOSIS AND SURGICAL TREATMENT JM Tew, SM Weil. University of Cincinnati, USA

Angiographically obscure vascular malformations are a common cause of spontaneous brainstem haemorrhage in young normotensive individuals. Since 1986 seven patients have presented to the authors with brainstem haematoma secondary to ruptured malformations. Five of the patients presented with progressive neurological dysfunction consistent with recurrent haemorrhage, one having undergone proton beam irradiation. Two additional patients had had one or two episodes of haemorrhage, but had recovered neurological function. Six of the malformations escaped angiographic detection while one case demonstrated multiple abnorma vessels in the early venous phase consistent with a venous angioma. Magnetic resonance imaging (MRI) demonstrated a characteristic morphology. Multiple haemorrhages of varying ages are common in brainstem vascular malformations. The ageing quality of haemoglobin permits accurate differentiation by MRI. In four cases the signal void of flowing blood in vessels was detected inside or adjacent to the haematoma. Age of haemorrhage on MRI correlated well with timing of clinical haemorrhage in all patients.

Five patients with recurrent haemorrhage and progressive neurological deficit had surgical extirpation of the haematoma and vascular malformation. The patients were operated on in a lateral oblique position via a midline suboccipital craniotomy and section of the inferior cerebellar vermis. Discolouration or distortion of the floor of the fourth ventricle either by haemorrhage or by the malformation itself was noted in all cases.

Histological evaluation of all cases demonstrated the malformations to be either cavernous or venous angiomas. Postoperative MRI has shown complete resection of the malformations. Of the five patients that have had surgery, three have returned to premorbid employment, while two are in an early recovery period and improving. There have been no episodes of recurrent haemorrhage. The two patients 
that have not had surgery are being carefully followed for progressive neurological deterioration which will document the need for surgical intervention.

In conclusion, MRI has facilitiated diagnosis and localisation of vascular malformations of the brainstem. The authors believe that radiosurgery for such malformations to prevent re-bleeding was associated with increased neurological deficit and treatment failure in some cases and recommended surgical extirpation for patients with progressive neurological deterioration due to recurrent haemorrhage from these lesions. The role of surgery and the natural history of these conditions was discussed.

HIGH DOSE NALOXONE AND CALCITONIN GENE RELATED PEPTIDE IN SUBARACHNOID HAEMORRHAGE

FG Johnston, BA Bell, JD Miller, D O'Shaughnessy. Atkinson Morley's Hospital, London; Edinburgh; Celltech Ltd, Slough

In 1981 the opiate antagonist naloxone was first reported to improve neurological deficits in two patients after subarachnoid haemorrhage (SAH), ${ }^{1}$ an effect confirmed in five of 14 patients given $2 \mathrm{mg}$ naloxone. ${ }^{2}$ In a double-blind placebo controlled study of 53 patients using $10 \mathrm{mg}$ naloxone, 12 improved and none were worse after naloxone on the Hunt Hess scale and 21 improved and 1 was worse on the modified Glasgow Coma Scale. $^{2}$ After normal saline injection, two patients improved and two were worse on the Hunt Hess scale, and eight improved and eight were worse on the MGCS. None of the five patients given the alkylparaben mixture (vasoactive vehicle for naloxone) responded. There were no changes in mean systemic blood pressure or arterial blood gases between placebo and naloxone groups. The beneficial effect of $10 \mathrm{mg}$ naloxone was significant and independent of clinical grade, blood on CT, age or aneurysm site.

The authors also reported their early experience with calcitonin gene related peptide whose existence was first predicted by molecular biology techniques and whose alpha form is widely distributed in nervous tissue and perivascular neural network. It is a vasodilator and in large doses reduces blood pressure. In rats CGRP reduces carotid vascular resistance at a dose which does not cause hypotension. ${ }^{3}$ The carotid vascular bed seems particularly sensitive to the peptide. Volunteer studies in man have elicited marked facial flushing and a feeling of fullness in the head with an increase in heart rate and a fall in blood pressure at high doses. ${ }^{4}$ The Doppler pulsatility index in the internal carotid and middle cerebral arteries is significantly increased and CGRP offers unique actions on the cerebral vasculature which may be therapeutically relevant to ischaemia after SAH.

Ten SAH patients who were neurologically impaired following aneurysm clipping had been given an escalating dose infusion of human alpha CGRP over 60 minutes up to a maximum dose of $1.15 \mu \mathrm{g} /$ min of active peptide. Eight of the 10 patients improved their MGCS, and abnormally high middle cerebral artery peak and mean velocities fell in one patient in whom Doppler measurements were made. Mean systemic blood pressure was marginally reduced at the highest infusion rates and facial flushing occurred in $80 \%$ of patients. The response rates of $40 \%$ to naloxone, and $80 \%$ to CGRP in these acute studies warrant further trials of CGRP infusion and oral administration of the naloxone analogue naltrexone during the initial weeks after SAH to determine the effect on long-term outcome.

1 Baskin DS, Hosobuchi Y. Naloxone reversal of ischaemic neurological deficits in man. Lancet 1981;ii:272-5.

2 Beel BA, Miller JP, Neto NGF, O'Neill P, Laughton LM. Effect of naloxone on deficits after aneurysmal subarachnoid haemorrhage. Neurosurg 1985;16:498-501.

3 Gardiner SM, Compton AM, Bennett T. Regional haemodynamic effects of calcitonin gene-related peptide. Am J Physiol 1989; 256:R322-38.

4 Gennari C, Fischer JA. Cardiovascular action of calcitron gene-related peptide in humans. Calcified Tissue International 1985;37:581-4.

INSULIN PROTECTS COGNITIVE FUNCTION IN CEREBRAL ISCHAEMIA

AJ Strong, JE Fairfield, M Snape, E Montiero, M Kirby, L Rossfield. Institute of Psychiatry, London

Maintained delivery of glucose to the brain in incomplete cerebral ischaemia is associated with adverse outcome, due to acidosis. Mild insulin-hypoglycaemia confers biochemical ${ }^{1}$ and electrophysiological ${ }^{2}$ protection: this study examined whether insulin also preserves function in a recovery stroke model.

Rats were trained on an 8-arm radial maze, and later underwent bilateral common carotid occlusion (snare) with hypotension. Treatment groups were: untreated sham (US, $\mathrm{n}=6)$, insulin-treated $(2 \mathrm{u} / \mathrm{kg}$ intraperitoneal) sham (TS, $n=6$ ), untreated ischaemic (UI, $\mathrm{n}=11)$ or insulin treated ischaemic (TI, $n=7)$. Sham animals unçwent the same surgical procedure. After weeks recovery, the maze task was repeatogd.

Four animals died in the UI and two in the TI groups (ns, all permutations). Refereflce memory (first entry into unbaited arms) Wh unaffected by ischaemia. However, in case of working memory (re-entry errors $\overline{2}$ way ANOVA indicated very significan ly worse performance in the UI group (p.< $0.0001)$, whereas TI rats were indistinguiş大 able from US/TS animals. Mean (SD) mmimum plasma glucose in TI animals was $\$ 1$ $\mathrm{mmol} / \mathrm{l}(0 \cdot 59)$. The minimum single value was $3.1 \mathrm{mmol} / \mathrm{l}$. In UI animals pre-lesß̧n plasma glucose was $7.9 \mathrm{mmol} / \mathrm{l}$ (SE 0.4) rose to $14.5(1 \cdot 1)$ immediately following occlusion and with hypotension rose to some $16 \mathrm{mmol} / \mathrm{l}$.

Insulin confers virtually complete funttional protection of working memory in the particular neuroanatomical system examined (hippocampus), and this can achieved at a level of hypoglycaemia whieh may be acceptable for use in man. The resulfs have important implications not only in relation to our understanding of ischaemic mechanisms, but also for brain protectionfin cardiothoracic and hypotensive anaestresta and in subarachnoid haemorrhage pagd $_{2}$ stroke. The feasibility of a similar regimsn in patients at risk of cerebral ischaemia showitd be explored.

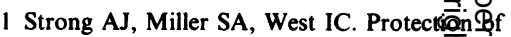
respiration of a crude mitochondrial preparation in cerebral ischaemia by contogl of blood glucose. J Neurol Neurosurg P $\$ 9$ chiatry 1985;48:450-4.

2 Robertson CS, Grossman RG. Protection against spinal cord ischaemia with insuli induced hypoglycaemia. Neurosurg 198., 67:739-44.

CAROTID ENDARTERECTOMY-DOES IT PREVENT STROKE?

DA Allcut, M Chakravorty, RP Senguptă. Newcastle

The role of surgery in the management cerebral ischaemia due to thromboembo fic disease is controversial and the benefit of carotid endarterectomy has yet to be proven beyond doubt. The authors reported their experience with 91 consecutive carogd endarterectomies performed on 83 patients by the senior author between 1975 a f d January 1987. Seventy-one patients we treated for transient ischaemic attacks a 12 patients had minor completed stroke. patients received medical treatmegat preoperatively, 67 had continued to be sympe 
tomatic despite medical treatment and 16 were selectively referred for surgery in addition to medical treatment. Eighty six carotid endarterectomies in 78 patients were completely uncomplicated. Two patients had permanent neurological deterioration and three died-two from ipsilateral stroke and one due to myocardial infarction. Hence, the overall operative mortality was $3 \cdot 3 \%$ and the combined peri-operative mortality and stroke rate was $5 \cdot 5 \%$.

Eighty patients were available for long term follow up of which full details were obtained for 77 . During a mean follow up period of $5 \cdot 2$ years, three patients experienced a major stroke affecting the cerebral hemisphere ipsilateral to the carotid endarterectomy. The incidence of stroke in this series over the mean follow up period was $-9.4 \%$, which includes the peri-operative strokes and deaths. Nine patients died during follow up, two due to stroke and seven as a result of myocardial ischaemia.

The results were discussed in relation to the natural history of carotid occlusive disease and compared with previous reports of surgical treatment

IMPAIRED CEREBRAL HAEMODYNAMICS IN INTERNAL CAROTID OCCLUSION

JM Derlon, G Bouvard, S Khoury, B Dupuy, MC Petit, F Viader, JP Thenint, JP Houtteville. Caen, France

In 75 patients in whom a unilateral or bilateral chronic internal carotid artery occlusion was demonstrated, the authors performed single photon haemodynamic assessment including a study of regional cerebral blood flow (inhalational 133Xenon clearance) and single photon emission computerised tomography of regional cerebral blood volume (with 99M Tc labelled erythrocytes). Intravenous acetazolamide was used to stress the cerebral circulation and assess its reserve capacity in some patients. Four haemodynamic patterns were demonstrated of which one was related to a poor haemodynamic reserve with severely impaired autoregulation. Theoretically, such a limited haemodynamic reserve should be associated with higher risk of delayed haemodynamic stroke distal to the occlusion.

This pattern of impaired reactivity was exhibited chronically by $24 \%$ of all patients and $35 \%$ of those who would have been eligible for randomisation in the extracran$\mathrm{ial} /$ intracranial bypass international study. Follow up of the 31 eligible patients with normal reactivity showed a very low risk of delayed ipsilateral stroke ( 1 case) or any stroke ( 1 case) over 21 months. Follow up of the 18 patients with impaired reactivity was indicative of a high risk of stroke in nonoperated patients but also of greater perioperative morbidity/mortality $(18 \%)$ in those who had bypass surgery.

Hence, amongst those patients who would have been considered as suitable for randomisation on clinical and radiological data only a few had impaired reactivity and would justify a bypass operation. However, the clinical benefit of surgery in patients with impaired reactivity must be weighed against the greater risk of perioperative morbidity and mortality.

\section{AGGRESSIVE MANAGEMENT OF SEVERE HEAD}

TRAUMA-TIME FOR REAPPRAISAL

RM Gibson, GC Stephenson. Leeds

"High technology" aggressive treatment of head injuries was introduced in Leeds a decade ago and involved the evacuation of mass lesions where present, ventilation on the intensive care unit, continuous measurement and control of intracranial pressure as well as measures to maintain the circulatory, renal and respiratory systems. Treatment continued until the patient died, or was declared brain dead, or improved sufficiently to be transferred to a neurosurgical ward. Audit of this treatment is timely, not only for fiscal and resource reasons but also on moral and ethical grounds. Between 1984 and 1987 , there were 187 patients who received such intensive treatment of whom 100 survived. A survival index was established with the following individual weighting factors: Age $0-40=0,41-60=1$, Over $60=$ 2. Pupils Unilateral unreactive 1 , Bilateral unreactive 4. Systolic, blood pressure less than or equal to $80 \mathrm{~mm}$ mercury $=4$. Glasgow Coma Score at presentation $=6-8$ 1, 3-5 2, extracranial injuries 3 (multiple, limb fractures, chest, laparotomy or positive peritoneal lavage); Intracranial pressure at time of admission to the intensive care unit and at 12 hours: Under $20=0,20-40 \mathrm{~mm}$ mercury $=2$, Over $40=4$. CT scan: intracerebral high density lesions 2 , intra and extracerebral high density lesions 4 .

Mean score in survivors-5.03 (SD) 2.35 (99\% confidence interval of the mean 4.50 to 5.56): non-survivors 11.69 (3.96) (confidence interval 10.72 to 12.66$) 99 \%$ confidence interval difference of the means 5.6 to 7.7 $(p<0.01)$. With a score over 14 only 3 out of 10,000 patients would survive.

This index was applied prospectively to 52 cases admitted during 1988 of whom 29 survived. The patients were divided into two groups 24 hours after admission - the certain to die group and the unable to predict group. All 10 patients entered into the certain to die group succumbed.

The authors concluded that there exists a group of severely head injured patients whose death can be predicted with a $100 \%$ accuracy at an early stage provided appropriate clinical, CT and intracranial pressure information is available. The authors proposed that a survival index score over 14,12 hours following admission, should lead to cessation of intensive care treatment.

\section{AUDIT OF MORTALITY IN A NEUROSURGICAL UNIT \\ P Barlow, B Jennett. Glasgow}

Special problems are posed by an audit of mortality in a neurosurgical unit. The majority of admissions are urgent or emergency, some patients are found shortly after admission to have a hopeless prognosis and many deaths occur without an operation having been performed and would not be included in a CEPOD-type audit. The challenge is to define and identify potentially avoidable factors that have contributed to, or caused, death in order that these may be discussed in a critical but non-judgemental atmosphere. This unit had previously reported on avoidable factors contributing to head injury deaths.

The present review concerned the deaths that were reported at the monthly mortality meetings of the Department during 1988. There were 131 deaths in the neurosurgical unit of whom 56 had operations. Forty-six potentially avoidable factors that contributed to, or were responsible for, death were identified in 38 patients. Treatmentlimiting decisions were recorded in the notes of 67 patients.

Such a regular, formal audit of deaths highlights shortcomings in management, educates junior staff and provides a forum for discussion of the problems of a more extensive audit. A wider audit might include independent judgement of performance, the consideration of deaths in neurosurgical patients after discharge from the unit, and the discussion of 'near miss' cases, or cases of avoidable morbidity. Extra resources would be necessary to address these additional issues.

DO CHILDREN WITH SEVERE CLOSED HEAD INJURY BENEFIT FROM INTENSIVE CARE? CGH West, R Kumar, EL Hall, DC Quirke, RJ Taylor. Salford

Between 1976 and 1987101 children aged 16 
years or under with major closed head injuries (Glasgow Coma Score 8 or less for six or more hours after injury) were admitted to the Royal Manchester Children's Hospital. Eight children were the victims of nonaccidental injury, and nine were aged 36 months or less at the time of their injury. Of the remaining 84 children, 27 were treated conservatively (general nursing care on the open ward, medication as seemed clinically appropriate), and 57 were treated intensively (intracranial pressure monitoring, diuretic therapy for measured intracranial hypertension, and hyper-ventilation).

The two groups were comparable in age and sex distribution, but there was a higher proportion of more severely injured patients in the intensively treated group. Nonetheless intensive treatment was associated with a lower mortality. The price paid is a higher proportion of children left with a moderate or severe disability as assessed on the Glasgow Outcome Scale, but there is no corresponding increase in those left in a persistent vegetative state. Of the 84 children comprising this study, 32 died. Most children died within 96 hours of injury regardless of whether or not they had received intensive care. Intensive therapy did not appear to needlessly prolong the misery and suffering of relatives when a fatal outcome was inevitable. If the child lived for seven days after injury, then the likelihood was that he would survive.

As expected, the children with moderate or severe disabilities remained in hospital longer than those with a good outcome, thereby further increasing the financial implications of intensive treatment.

Of the 52 survivors, 50 were recalled and examined independently by neurosurgeons and clinical psychologists. Their findings were kept quite separate until completion of the study when all the data were pooled. The neurological assessment comprised a neurological examination and assignment of a score on the Glasgow Outcome Scale after further discussion with the patient and his family. The psychological assessment addressed intelligence, verbal and visual memory, the ability to handle information, and social re-integration. Tests particularly sensitive to cognitive impairment were chosen. When applied to those children who had been deemed by the neurosurgeons as having made a "good recovery" on the Outcome Scale, it was clear that this assessment is too crude; many children in this group have severe impairment of intelligence, learning ability and memory and many have great difficulty in re-integrating into society. Nonetheless, when compared with conservative treatment, intensive care would seem to lessen this cognitive impairment.

TRANSIENT TRAUMATIC CORTICAL BLINDNESS IN CHILDREN-A TYPE OF CLASSICAL

MIGRAINE IN CHILDHOOD

P Eldridge, JAG Punt, S Clarke, NJ Smith. Nottingham

This condition is poorly recognised with an incidence in the literature of about $0.4 \%$ but the authors prospectively had identified 23 cases $(0.7 \%$ of casualty admissions for head trauma under the age of 12). The incidence was lower in the summer months. Typical features include a blow to the occipital region of the skull following a short fall on to a hard surface without loss of consciousness but the child may be dazed. There is a latent period of up to 45 minutes with no visual problems followed by gradual blurring and finally loss of vision during which the child may describe the world as appearing white. After a variable period of between $30 \mathrm{~min}$ utes and 12 hours there is full recovery of vision often described as reappearing in the centre of the visual field. Other symptoms may include confusion, distress, unsteadiness, headache, drowsiness, nausea and vomiting. The authors stress that the child may not complain of visual difficulty $(30 \%$ in this series)

The authors review of their 29 patients (six retrospective and 23 prospective) confirmed that this syndrome may be a form of classical migraine - history of migraine $(17 \%)$, family history of first degree relative with migraine and visual aura $(65 \%)$, time course and latent period matches that of migraine, $5 / 6$ children studied had shown EEG changes similar to those shown in classical migraine and described experimentally in animals during spreading cortical depression and EEG mapping showed an occipital distribution of slow wave activity maximal at 24 hours and taking one week to resolve. Diagnosis is clinical and carries an excellent prognosis.

EXTRADURAL HAEMATOMA IN INFANTS

JRS Leggate, N Lopez-Ramos, L Genitori, G Lena, M Choux. Marseille, France and Edinburgh

From 219 children with extradural haematoma (EDH) treated between 1960 and 1988, a series of 40 cases under two years of age was presented. Twenty five $(63 \%)$ were male, $15(37 \%)$ female. The patients were divided into three groups; Group A (11 pts), less than six months old; Group B (16 pts), seven to 12 months; Group C (13 pts) 13 months to two years. Falls less than 1 meze accounted for $16(40 \%)$, falls from more than 1 metre were seen in $12(30 \%)$. Falls when walking occurred in seven $(17 \cdot 5 \%)$. T\$o EDH were treated following obstetfic trauma. Road traffic accidents accounted for only three $(7.5 \%)$ cases. There were no caşes of non-accidental injury. Nine cases (22.5\%) suffered immediate loss of consciousness lucid interval was identified in 30 cases, in. 5 it was longer than 24 hours. Drowsins $(60 \%)$ and delayed vomiting ( $45 \%)$ were the most important symptoms. Anaemia detected in $19(47 \cdot 5 \%) .36(90 \%)$ had abnormal X-ray with either a fracture or suture separation or both. Thirty (75\%) EDH were parietal, temporal or tempof̧parietal. Two were located in the posterfor fossa. There were no frontal EDH. All we户e treated by craniotomy. Bleeding was from the middle meningeal artery in $17(42.5 \%)$, the bone in $11(27 \cdot 5 \%)$, the dural surface 9 n three $(7 \cdot 5 \%)$ and unknown in nine $(22 \cdot 5 \%)$. There were five deaths $(12 \cdot 5 \%)$ and six $(15 \%)$ had sequelae after surgery. The authors concluded that under the age of 6 months, a prolonged lucid interval, droustness and a skull fracture were the thast prominent clinical features. The comfichtion of a skull fracture, with anaempa, delayed vomiting or drowsiness shoulole to immediate CT.

THE RELATIONSHIP OF CEREBRAL BLOOD R VELOCITY TO THE INTRACRANIAL PRESSLEB INFANTS AND NEWBORNS WITH PROGRESTVE. HYDROCEPHALUS AJW Steers, M Hendry, M Thambyay RA Minns. Edinburgh

The authors reported their investigation the relationship between cerebral arte $\overline{\text { a }}$ al blood flow velocity as measured by the resistance index and intracranial pressurean 27 subjects who were all infants with hydrocephalus of variable aetiology. Bhe technique of transfontanelle transcranial Doppler Ultrasound was used to measyre peak (systolic) and diastolic blood fleiw velocity in the anterior and middle cerebal arteries and to calculate a resistance in ax $(\mathrm{RI}=(\mathrm{S}-\mathrm{D}) / \mathrm{S})$ for flow velocity in exeh subject. Transfontanelle ultrasound scänning also facilitated the assessment of degigee of ventricular dilatation. In each subject paired measurements of ventricular CSF pressure as measured by a non-displacement method using a strain gauge connected viaten anterior fontanelle puncture or a CSF access device was compared with the resistance index. In 15 cases, Doppler and ICP assessment was performed both before and affer 
the withdrawal of a known volume of CSF. ICP values in the study group ranged from 4 $40 \mathrm{~mm} \mathrm{Hg}$. The study concluded that there was a positive correlation between the intracranial pressure and cerebral blood flow velocity and between the cerebral perfusion pressure and cerebral blood flow velocity. However, there was a poor correlation between cerebral blood flow velocity and ventriculomegaly. Reduction of intracranial pressure by the removal of ventricular CSF achieved improvement in the cerebral blood flow velocity but there was no consistent linear or exponential volume/flow velocity relationship.

\section{VANCOMYCIN PHARMACOKINETICS IN} HYDROCEPHALIC SHUNT PROPHYLAXIS PD Le Roux, MA Howard, DL Silbergeld, HR Winn. Seattle, USA

Thirteen patients received ventriculoperitoneal shunts for hydrocephalus. One hour before surgery each patient received a standard dose of $1 \mathrm{~g}$ of Vancomycin in 250 $\mathrm{ml}$ saline infused over 30 minutes. Samples of CSF and blood were obtained one hour later and Vancomycin levels assayed by fluorescence polarisation immunoassay.

There were five females and eight males with an average age of 44.5 years. All had normal renal function and the average weight was $72.6 \mathrm{kgs}$. Nine patients had suffered subarachnoid haemorrhage, two trauma and one each had normal pressure hydrocephalus and tumour associated hydrocephalus.

CSF Vancomycin levels at one hour ranged from $0 \cdot 1-1 \cdot 5 \mu \mathrm{g} / \mathrm{ml}(0 \cdot 8,0 \cdot 36)$. Blood Vancomycin levels ranged from $9 \cdot 1-38 \cdot 7 \mu \mathrm{g} /$ $\mathrm{ml}(21.9,8.97)$. There was no significant difference in CSF Vancomycin levels if CSF protein was greater than $0.8 \mathrm{mg} / \mathrm{dl}(\mathrm{p}<0.1)$ nor blood Vancomycin levels in the therapeutic range $(p<0.5)$. No patient had evidence of shunt infection at six month follow-up. The MIC of Vancomycin for Staphylococci is $1 \cdot 5-3 \cdot 1$. Bactericidal levels of 5-8 MIC are needed to kill organisms. It appears that the efficacy of Vancomycin prophylaxis in shunt procedures may be more related to adequate tissue levels being achieved before surgery than to CSF penetrance.

SHUNT INFECTIONS IN INFANTS AND CHILDREN FOLLOWING SHUNT INSERTION

AR Walsh, A Sofat, RD Hayward, DN Grant. Hospital for Sick Children, Great Ormond Street, London
Improved survival of premature infants has led to an increased number of referrals of low weight infants for control of hydrocephalus secondary to intraventricular haemorrhage (1VH). Shunt infections continue to represent a considerable risk of morbidity in these children. In a one year period (1984/5) the overall infection rate was $12 \%(13 / 110$ shunt procedures). Age significantly affected the risk of infection, being $21 \%$ in children less than six months and only $2 \%$ in children over six months of age $(0.01>p>0.001)$. When allowance for age was made, primary insertion carried the same infection risk as revision. Primary shunt insertions (116 of 126) over a two year period (1985/6) were then analysed in detail. Children had a high risk of infection in all age groups less than six months of age and within this age group those children born with a gestational age of less than 32 weeks had a similar infection rate $(23 \%)$ as those born at full term $(25 \%)$ suggesting that age from birth rather than developmental age is the dominant risk factor. The status of the surgeon (consultant or surgeon in training) and the type of shunt inserted (paediatric Hakim or Holter + Rickham reservoir) did not significantly affect the risk of infection. It had not been possible to analyse the effect of duration of operation. When considering future trials of prophylaxis against infection particular attention to the high risk group must be given.

\section{MANAGEMENT OF ENCEPHALOCOELE}

AD Hockley, JG Hamilton, JH Goldin, MJ Wake. Birmingham

The aim of surgery is to restore normal anatomy with repair of the defective dura, bone and skin and such surgery may usually be elective but in some instances may be urgent and life-saving-for very thin skin cover or a ruptured lesion (loss of CSF, haemorrhage and infection), airway obstruction from an intranasal encephalocele, eyeball displacement causing impairment of normal development of vision and recurrent meningitis or CSF leak after erroneous biopsy of a nasal 'polyp'. Prognosis depends on the location of the lesion, its content, size and associated anomalies. Occipital lesions do less well than frontal or basal. Anterior lesions are usually associated with normal development even if the cosmetic deformity is grotesque. Encephalocoeles have a higher mortality and lower IQ than meningocoeles as did very large lesions or the presence of a microcephalic head. Hydrocephalus complicates $50 \%$ of occipital encephalocoeles which contain cerebellar tissue. The authors presented their experience (1979-89) of 20 occipital lesions and 10 anterior lesions (eight sincipital and two basal). Of the 20 occipital lesions, follow up continued on 17 (five meningocoeles and 12 encephalocoeles). The three patients with meningocoeles without hydrocephalus had developed normally whereas two patients with hydrocephalus were mentally and physically retarded. Of six encephalocoeles without hydrocephalus, 5 had developed normally and of 6 patients with hydrocephalus, 2 had died, 2 had remained very poor and only 2 were normal. Of the anterior lesions, the results were more satisfactory without any operative mortality although in one patient cerebral oedema occurred in the post operative period leading to residual intellectual impairment. Surgical repair of these lesions is usually straightforward but complex anterior and basal cases are probably best managed by a cranio-facial team approach.

HAS MODERN MANAGEMENT IMPROVED THE OUTCOME OF INFANTS WITH INTRACRANIAL TUMOUR?

R Kumar, RAC Jones, H Tekkok. Salford

Records of 92 children in the first eighteen months of life with an intracranial tumour who were registered with the Manchester Children's Tumour Registry during the period 1953-87 were reviewed. Introduction of computed tomography to the region in 1977 improved the diagnostic accuracy and led to early diagnosis. The pre and postoperative CT were balanced for age, sex, distribution and histology (85\% malignant in both periods). Introduction of CT coincided with more refined radiotherapy and improved chemotherapy. The surgical attitude became more aggressive. Early treatment mortality was reduced from $38 \%$ to $16 \%$ and CT had reduced the number of operations performed for biopsy alone. All survivors were available for follow up. Survival was improved for the first six years postoperatively but long term follow up did not suggest an improvement in longer term survival nor in the quality of life.

EPIDERMOID AND CHOLESTEROL CYSTS IN THE APEX OF THE PETROUS BONE

JC Benjamin, TT King, AW Morrison. London Hospital, London

Cystic lesions in the petrous apex are usually either epidermoid or cholesterol cysts. Their pathogenesis remains unclear but experimental and clinical evidence supports the theory that epidermoids are congenital 
and cholesterol cysts acquired lesions. Thirteen cases have been encountered amongst a series of more than 500 cerebellopontine angle tumours over a period of 20 years. Such lesions may be symptomless for many years but then present with progressive cranial nerve palsies or breach of the CSF pathways resulting in meningitis or a CSF leak. High resolution CT scanning and magnetic resonance imaging have facilitated their diagnosis. Surgical treatment was inadvisable unless patients were symptomatic when the authors recommended early surgery especially once hearing loss, hemifacial spasm or facial weakness appeared. Recovery of cranial nerve function was unlikely to be satisfactory if deafness or facial paralysis was severe or longstanding. The authors found that the posterior fossa approach was the only reliable method available for draining cysts whilst preserving hearing, though with appropriate medial extension the trans-sphenoidal approach might be successful.

The results of surgical treatment differ between the two groups, with limited benefit obtained by patients with epidermoid cysts (seven patients) compared to those with cholesterol cysts (five of six improved). In all, 10 out of 13 patients were helped by surgery. One patient with a cholesterol cyst developed chemical meningitis and hydrocephalus.

\section{SURGICAL MANAGEMENT OF HAEMANGIOBLASTOMA OF THE SPINAL CORD: EXPERIENCE OF 18 CASES}

L Symon, T Murota. The National Hospital, London

Haemangioblastomas of the spinal cord are uncommon tumours in comparison with those of the posterior fossa, either as solitary lesions or as part of the von Hippel-Lindau complex. Eighteen cases of spinal haemangioblastoma had been operated on between 1972 and December 1988 and the clinical and radiological data of these patients were reviewed.

There was a preponderance of males (M:F 1.6:1) and 15 cases had the onset of spinal symptoms before their forties. There were 19 intradural and two extradural tumours. The tumour was accompanied by a cyst in nine cases $(50 \%)$. Six cases of Lindau's disease including three cases of multiple spinal tumours were found. The symptoms improved in 13 cases postoperatively $(72 \cdot 2 \%)$, were unchanged in two cases and were aggravated in three cases. Magnetic resonance imaging (MRI) with Gadoliniumdiethylene-triamine-pentaacetic acid (Gd-
DTPA) enhancement was found to be a useful tool for precise localisation of the tumour and differentiation of any accompanying cyst. Spinal angiography was recommended preoperatively. Care should be taken to devascularise the tumour from outside and not enter it before removal. The availability of magnetic resonance facilitated extended family studies but some asymptomatic individuals reacted badly when told they had multiple lesions that might give trouble in the future.

TRANSFORMATION OF ENDOTHELIAL CELLS BY GLIOMA-CONDITIONED MEDIUM

D Silbergeld, F Ali-Osman. Department of Neurological Surgery, Seattle, USA

Abnormal neovascularity with endothelial cell proliferation and subsequent alteration of the normal blood-brain barrier is a hallmark of malignant astrocytomas. Although extensive transformation changes of the astrocytic component of these tumours have been well documented, concomitant transformational changes in the endothelial cell component have not been investigated previously.

Cell cultures of normal aortic arch endothelial cells and astrocytoma cultures from a rat glioblastoma cell line were characterised with light and electron microscopy, flow cytometric analysis of DNA and RNA content and tritiated thymidine quantification of DNA synthesis. Astrocytoma conditioned medium from confluent astrocytoma cell cultures was added in varying concentrations to subconfluent endothelial cell cultures. After twenty four hours exposure to conditioned medium, endothelial cells were again characterised with microscopy, flow cytometry, and thymidine labelling index. Although the endothelial cells were microscopically unchanged, RNA and DNA content were dramatically altered indicating that the endothelial cells had undergone transformational changes.

Because tumour endothelial cells are a major consituent of the tumour-blood-brain barrier, a better understanding of the biology of these cells is important for designing and delivering chemotherapeutic agents. Furthermore, tumour endothelial cells may be an important target for anti-neoplastic agents in the future.

SPECIFIC TARGETING OF MONOCLONAL ANTIBODIES TO MALIGNANT BRAIN TUMOURS: THE PRESENT STATUS

RP Moseley,* MR Zalutsky, † DD Bigner, $†$ HB Coakham.*
Carolina, $†$ USA

Bristol* and

No色h

With the development of monoclofal antibody technology, antibody targeted delivery of radiation to brain tumours become an attractive concept. For exame, intravascular administration of radioiod ated anti-tenascin monoclonal antibsy $81 \mathrm{C6}$ has been shown to produce. a therapeutic response in rodents bear human glioma xenografts.'

$\stackrel{4}{\rightarrow}$

In order to evaluate the pharmacokine of I-131 labelled $81 \mathrm{C} 6$ antibody in human patients with intracranial tumours we administered 5-50 milligrams of Ilabelled 81C6 concurrently with approximately 1 milligram of I- 125 labelled $45 \cdot 6$, an isotype matched control monoclo $\overrightarrow{a l}$ antibody. Gamma camera images revealed. increased uptake of I-131 in tumour siess. Biopsy specimens of tumour and nornal tissues were examined histologically and For radioactivity content, enabling quantitatịon of immunoconjugate uptake. Tumour গुo normal brain ratios of $81 \mathrm{C} 6$ binding were as high as 200:1 in some biopsies. Localisat O indices for tissues were obtained by no ising 81C6 content to that of the coint antibody and correcting for differencesain plasma concentration of immunoconjugates. This parameter reflects specific imeruological binding and values of approximately 1 were obtained for normal tissoeson contrast to up to 5 for tumour. This gity demonstrates specific tumour bindie $81 \mathrm{C} 6$ in patients with intracranial malighancies.

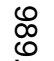

1 Lee Y, Bullard DE, et al. Cancer Res 1988; 48:2904-10.

A RELOCATABLE STEREOTACTIC FRAME SS Gill, DGT Thomas. The National Hoopital, Queen Square, London

A stereotactic head frame had been developed which was accurately relocatable over an extended period, was non-invasipye and yet rigidly fixed relative to the patients skull. This was achieved by using the patie upper dentitian and hard palate as the prime site for locating the frame on the hegd. Patient studies had shown that the accuracy of relocation was within $1 \mathrm{~mm}$ for any pomt in the head. The maximum displacement of thalamic targets measured in nine patiegts after relocation of the frame was $0.5 \mathrm{~mm}$. The mean displacement was $0.25 \mathrm{~mm}$ (St) $0.3 \mathrm{~mm}$ ( \pm 2 standard deviations).

The frame has a number of applicatiofs. Firstly it enables a series of investigations to 
be performed in stereotactic space at times convenient to the surgeon and radiologist, allowing surgery to be planned and performed at a later date. Secondly, it enables stereotactic procedures to be repeated without the need to redefine targets by repeated investigation. This has particular application to fractionated stereotactic radiotherapy using a linear accelerator. Thirdly, its non invasive relocation enables one to monitor pathology objectively over an extended period with good patient compliance. Finally, the compatibility of the frame with a variety of biomedical imagers facilitates the precise matching of different imaging modalities. The value of integrating this information has great potential in that it provides the means for objectively studying the functional information provided by -Positron Emission Tomography in the anatomical framework provided by Computer Tomography and Magnetic Resonance Imaging. Each of these applications was illustrated with clinical examples.

\section{CT GUIDED STEREOTAXIC CRANIOTOMY AND INTRAOPERATIVE LOCALISATION OF INTRACEREBRAL LESIONS HI Sabin, IR Whittle. Edinburgh}

A major problem facing the surgical neurooncologist is locating small lesions within the brain. Inappropriate placement of the craniotomy flap or corticotomy incision can lead to suboptimal surgery or postoperative neurological deficits. The authors described a simple technique, utilising the CT compatible BRW stereotaxic apparatus, that enables both optimal placement of craniotomy flaps and precise intraoperative localisation of intracerebral lesions.

Appropriate craniotomy and target points were selected from CT performed with the BRW localiser ring. The coordinates for these points were computed and the craniotomy or trephine performed and lesion localised. The four point BRW base ring served as both a head fixator and an attachment for the stereotaxic arc system. The Budde-Halo or Yasargil self retaining retractor systems could be attached to the base ring, and, after the lesion had been localised. the stereotaxic arc system was rotated to serve as a hand rest. This method of stereotaxic microsurgical craniotomy had been used in 12 patients aged 21-66 years with cerebral neoplasms (four glioblastoma, three astrocytoma/oligodendroglioma, 3 metastases, 1 meningioma and 1 supratentorial hemangioblastoma). These lesions had either been removed or debulked from eloquent areas of the brain without causing deficits or postoperative infection.

A LOW COST MODIFICATION OF AN OLD LEKSELL STEREOTACTIC FRAME TO ALLOW CT GUIDED STEREOTAXY

A Sofat, AP Jones, CHG Davis, NT Gurusinghe, S Denton. Preston and Christie Hospital and Holt Radium Institute, Manchester

Whilst most Neurological Centres possess one of the standard stereotactic frames, the transition to CT guided stereotaxy can be inhibited by the high cost of the commercial CT compatible frames. The authors had devised a modification to an old Leksell frame to allow its use with CT scanning. The approach adopted was to fix a pair of fiducial plates to the sides of the existing frame. This system was designed so that a single pair of plates would allow computation of the target's frame coordinate irrespective of the angular orientation of the frame. The modifications were non specific to any particular CT scanner. The calculation of the frame coordinates of the nominated target was made using simple trigonometry. The calculations were performed using a programme on a micro computer. The frame had been used successfully to localise lesions in 11 patients. The modification placed no extra constraints on patient positioning and the design of the fiducial system made the modifications more versatile than the present commercial Leksell CT adaptations.

\section{A SIMPLE CT STEREOTACTIC SYSTEM \\ JE Dervin, J Miles, Liverpool}

Techniques which represent a true three dimensional solution to the problems of CT stereotaxis (freedom from constraints regarding the orientation of the patient stereotactic instrument/CT scanner) rely on relatively complicated mathematical procedures.' A new method had been devised which owed its origins to an analogue (model) technique ${ }^{2}$ that permitted the development of a simple mathematical (three-dimensional) solution to the problems of CT linked stereotaxis.

A stereotactic instrument based on this system had been in use at the Walton Hospital, Liverpool for two and a half years. The compact size of this instrument design would facilitate its use with MR given the necessary material modifications such as replacement of the carbon fibre fiducials with hollow plastic tubes containing material returning high signal on MR (vegetable oil) and titan- ium for the fixation pins. The use of a carbon fibre base ring avoids the problems of eddy currents, and the necessity to manufacture a split metallic base ring is therefore eliminated. A mathematical analysis has been derived to allow the system to be used for functional stereotaxis. A simple visual alignment system to allow the stereotactic instrument to be used for CT linked radiosurgery with the linear accelerator is currently under development.

1 Brown RA. J Neurosurg 1979;50:715-20.

2 Dervin JE, Miles JB. Neurochirugia 1984;27: $162-5$

ROUND TABLE DISCUSSION-STEREOTACTIC LOCALISATION IN NEUROSURGERY J Miles, DGT Thomas, IR Whittle, CHG Davies

Discussion concentrated on the relative merits of various systems with particular emphasis on matching the sophistication of the system to the clinical problem. The Medico-legal implications of adaptation of existing frames was highlighted together with the need to define any system's mechanical and imaging precision. Other factors included portability, compatability with magnetic resonance, and a linear accelerator and accessibility for craniotomy and epilepsy surgery.

SPONTANEOUS DISSECTION OF THE CERVICAL INTERNAL CAROTID ARTERY: THE ROLE OF DOPPLER STUDIES AND CONSERVATIVE MANAGEMENT

MSM Eljamel, PRD Humphery, MDM Shaw. Liverpool

Spontaneous internal carotid artery dissection is more common than previously thought. Early reports associated this condition with a high morbidity and mortality; however, recent studies suggested that most patients who survive the initial episode usually do well. The diagnosis is based on the demonstration of tapered narrowing of the internal carotid artery "string sign" on angiography. However, it is impracticable and usually impossible given an angiogram to every patient presenting with stroke. Doppler ultrasound screening of young patients presenting with stroke to the Mersey Regional Department of Neurosciences, identified ten such patients with reduced common and internal carotid artery blood flow without any evidence of significant atheroma at the carotid bifurcation.

Eight of these patients, on angiography, proved to have a dissection of the internal 
carotid artery. Five males and three females, 14-55 years of age, all had focal neurological signs, three with TIA's, three with stuttering strokes and two with completed strokes. All of them were managed conservatively; seven received anticoagulants arresting any further neurological symptoms without any serious neurological complications. Those who had anticoagulant therapy started within the first three weeks of the ictus had a good outcome and showed improvement of the common and internal carotid blood flow on subsequent Doppler studies.

\section{OCULAR MICROTREMOR AND BRAIN STEM FUNCTION \\ G Bolger, G Fry, D Coakley, J Philips,} N Sheahan, J Malone. Dublin

Ocular microtremor (OMT) is a constant, physiological high frequency tremor of the eye. Since eye movement is generated at brainstem level, it has been postulated that OMT could be a useful indicator of brain stem dysfunction' The authors had developed a highly portable and accurate method of recording OMT which unlike previous systems had a flat frequency response between 20 and $200 \mathrm{~Hz}$. Using this method the frequency of OMT was compared in normal (11), comatose (11) and brain dead subjects (13). Non medical comatose subjects were selected from serial admissions to the Neurosurgical ICU. Controls were age matched. All records were analysed blind.

The frequency of OMT in the normal group was $83 \pm 6.5 \mathrm{SD} \mathrm{Hz}$, while that of the comatose group was $45 \pm(\mathrm{SD}) 16$. $(\mathrm{t}=7 \cdot 4$, $\mathrm{p}<0.001)$. Ten patients with a diagnosis of brain death had no OMT activity. However, in one subject, OMT activity persisted for 72 hours following the diagnosis prior to cardiac arrest. Two patients initially in the coma group deteriorated to a state of brain death. In both cases OMT activity, though present on admission, was not present at the time of diagnosis of brain death. Serial recordings on seven patients indicate that, for any individual, improvement or deterioration in clinical state is reflected in a parallel change in OMT activity.

1 Coakley D. Minute Eye Movement and Brain Stem Function 1983, CRC Press, Florida, USA.

MAGNETIC STIMULATION IN PATIENTS WITH CERVICAL SPONDYLOSIS

RJ Laing, DJ Jaskolski, JA Jarratt, J Jakubowski. Sheffield
Magnetic stimulation is a new, painless method of assessing conduction in central and peripheral motor pathways.'

Forty-two patients with cervical spondylosis were divided into three groups according to clinical and radiological findings, namely: spinal cord compression, radiculopathy, and clinical radiculopathy but negative radiology. Motor conduction time (MCT) between head and neck was measured in pathways to abductor digiti minimi (ADM) in all patients. MCT between head and neck in pathways to biceps brachii (BB), and between head and lumbar region in pathways to abductor hallucis (AH) was also measured in some.

In patients with cord compression, the mean MCT to ADM (13.4 ms) was significantly longer than normal $(10.0 \mathrm{~ms} ; \mathrm{p}<$ $0.01)$. The mean MCT to AH (24.1 ms) was also significantly longer than normal $(18.8$ $\mathrm{ms} ; \mathrm{p}<0.02$ ), but the mean MCT to BB $(8.3 \mathrm{~ms})$ was not significantly different from control data (8.6 ms). Abnormalities were present if cord compression was at $\mathrm{C} 3 / 4$ or $\mathrm{C} 4 / 5$, but were not found when compression did not extend above $\mathrm{C} 5 / 6$. In the other two groups, mean MCTs were not significantly different from control data. Fourteen of the patients were also studied post-operatively three months or longer after surgery. In those with abnormal MCTs before surgery and who improved clinically afterwards, the post-operative mean MCT in pathways to ADM (12.9 ms) was significantly less than the pre-operative value $(20.2 \mathrm{~ms} ; \mathrm{p}<0.05)$. In patients who were unchanged or worse, there was a trend in the MCTs which followed the clinical outcome. The technique does not reveal clinically silent lesions but quantifies established clinical lesions and facilitates objective evaluation of outcome.

\section{Barker AT, Jalinous R, Freeston IL. Lancet 1985;i:1106-7.}

MICROVASCULAR DECOMPRESSION FOR HEMIFACIAL SPASM: COMPLICATIONS AND LONG TERM RESULTS

R Illingworth, London and J Jakubowski, Sheffield

The results were presented from two neurosurgeons of microvascular decompression for hemifacial spasm performed in 69 patients since 1978 . With the patients in the lateral position the root exit zone of the VIIth nerve had been explored through a small retromastoid craniectomy. Arterial cross-compression was found in 65 and veins in four. The arteries were dissected free and cottonoid interposed or the arteries held away with cottonoid slings. Hemifaçal spasm often persisted postoperatively formp to $\mathbf{4 8}$ hours and in seven patients for upfo seven months. One patient relapsed and as re-operated and there was one delayed \&ut transient relapse. Sixty one patients ore under follow up and are free of hemifacal spasm between nine and 117 months (me 44) later, three were lost to follow-up witun two years and five after two years. All were free of hemifacial spasm at last contact. $\underset{\overrightarrow{\vec{\omega}}}{\overrightarrow{\vec{p}}}$

There were no deaths or major neurological postoperative complications. Ipsilateral deafness was complete in cases and partial in five. There were 3 temporary facial palsies (delayed in six) 速d two permanent (one partial), temporary vertigo in two and one each of meningitis and CSF leak. The procedure is effective $\overline{\text { on }}$ producing long term relief of symptoms. Cranial nerve complications remain $\vec{\omega}_{a}$ problem but lessen as experience increases. General discussion suggested that Botulinum toxin injections of the facial nerve or mechanical prodding through the midfde ear gave less permanent results particularty in the refractory sub-group than did microvascular decompression.

IS INTRA-OPERATIVE ULTRASOUND OF VA IN BRITISH NEUROSURGICAL PRACTICE? ত JC Sutcliffe, RDE Battersby. Sheffield

The authors described their initial six mand experience of intra-operative ultrassind (IOUS) and outlined the techniques used for localisation and biopsy of intracrarual lesions. A small $(3 \mathrm{~cm} \times 2 \mathrm{~cm})$ craniectomi/ craniotomy was required for IOUS and gle authors stressed that there was a learn curve with its use. A preliminary prospective study comparing IOUS to freehand biopsy or aspiration of supratentorial mass lesiक्षिis revealed:

\begin{tabular}{|c|c|c|}
\hline & $\begin{array}{l}\text { Freehand } \\
\text { (control) } \\
\text { (10) }\end{array}$ & $\begin{array}{l}\text { IOUS } \\
\text { (10) }\end{array}$ \\
\hline $\begin{array}{l}\text { Operating time (min) } \\
\text { Specimen adequacy \% } \\
\text { Complications }\end{array}$ & $\begin{array}{l}26(15-40) \\
100 \\
1\end{array}$ & $\begin{array}{l}48(15-90 \pm \\
100 \\
0\end{array}$ \\
\hline
\end{tabular}

IOUS detects immediate haemorrhagic coinplications, even when these do not reagh clinical significance. Since the study commenced some patients not included in the study have been referred specifically IOUS guided biopsy in lieu of a CT guifed stereotactic procedure.

The authors concluded that IOUS hasa. definite place in operative neurosurgery. N 
A STUDY OF CLINICAL MANAGEMENT AFTER MAGNETIC RESONANCE IMAGING IN SUSPECTED CERVICAL SPONDYLOTIC MYELOPATHY P Statham, D Hadley, P Macpherson, E Teasdale, R Johnston, I Bone, G Teasdale. Glasgow

The potential of magnetic resonance (MR) imaging to replace myelography with or without computed tomography (CT) in 106 patients with suspected cervical spondylotic myelopathy had been assessed prospectively, using a Picker $0 \cdot 15$ Tesla resistive imager. Patients with a firm clinical diagnosis of radiculopathy (without myelopathy), multiple sclerosis, tumour or syrinx were excluded.

At the clinician's request, 20 patients had a second investigation (myelogram 18, CT/ myelography 2), after $M R$. In nine the second investigation was performed to exclude pathology in the thoracic or lumbar spine, and in eleven others to obtain more specific information about the cervical region. The diagnosis was changed in only four, and management was not affected in any case. Thirty eight patients underwent a subsequent operation. In $34 \mathrm{MR}$ was the only imaging technique used. In the other four patients, the diagnosis and level of operation were not changed as a result of the second investigation. Four patients were unable to undergo MR because of claustrophobia (three), or the presence of an aneurysm clip (one).

In conclusion, MR gives adequate information for diagnosis and surgical treatment of patients with suspected cervical spondylotic myelopathy. It has the advantage of being non invasive, involves no exposure to ionising irradiation, and can be performed as an outpatient.

\section{HIGH DEFINITION CT SCANNING IN LUMBAR} DISC SURGERY

MGJ O'Sullivan, TF Buckley, M Hickey, D Ryder. Cork

The introduction of computerised tomography (CT) for the diagnosis of prolapsed lumbar discs' has met with disagreement over its value. ${ }^{2.3}$ In August 1988, Cork acquired an IGE CT Pace scanner capable of $0.45 \mathrm{~mm}$ resolution. The scans obtained were of such impressive quality that the authors wished to evaluate its accuracy in the diagnosis of prolapsed lumbar disc in the initial 25 cases-those with spinal stenosis and lateral recess stenosis were excluded. In a prospective study, the scan was interpreted preoperatively by the operating surgeon with full knowledge of the clinical syndrome and the official neuroradiological report and then correlated with the operative findings: Correct $22(88 \%)$; equivocal $2(8 \%)$; misinterpreted $1(4 \%)$.

In the retrospective study, the scans were interpreted postoperatively in an independent blind fashion by two neuroradiologists (Ni) with one neurosurgeon (NS) and correlated with the operative findings.

\begin{tabular}{llll}
\hline & Correct & Incorrect & Equivocal \\
NR1 & $24(96 \%)$ & 0 & 1 \\
NR2 & $24(96 \%)$ & 1 & 0 \\
NS & $21(84 \%)$ & 3 & 1 \\
\hline
\end{tabular}

There were no negative explorations. Plain radiographs were not universally performed before CT scanning and resulted in difficulty with the interpretation of levels in three cases. Hard copy scans were of variable quality and depended on the 'windows' used during developing. Hence recent advances in CT scanning results in a high level of accuracy in the diagnosis of prolapsed lumbar discs provided the patients are very carefully selected but unsuspected lesions may be missed. Plain radiographs should always be available for the correct interpretation of levels.

1 Williams AL, Haughton VM, Syvertsen A. Radiology 1980;135:95-9.

2 Bell GR, Rothman RH, Booth RE. Spine 1984;9:552-6.

3 Moufarrij NA, Hardy RW, Weinstein MA. Neurosurgery 1983;12:184-8.

INTRADURAL BENIGN TUMOURS LYING

ANTERIOR TO THE SPINAL CORD-THE

LATERAL APPROACH TO SURGICAL EXCISION

S Young, J Singh, SA O'Laoire. Dublin

Anteriorly placed spinal meningiomas and neurofibromas displacing the spinal cord posteriorly present special difficulties for surgical removal. Rather like thoracic disc protrusions they are often hard and buried in the cord anteriorly. Excision of such lesions by the standard posterior laminectomy approach is associated, as in thoracic disc surgery, with a high incidence of postoperative neurological deterioration. It is more logical to approach these lesions anteriorly. The authors presented a series of 8 cases of wholly anteriorly placed tumours, seven meningiomas and one neurofibroma, which were excised totally without operative disturbance of the spinal cord. Access was by hemilaminectomy and pediculectomy. Dural opening was lateral. The tumours were excised by devascularisation of the tumours as a first step using laser or bipolar coagulator, followed by shrinkage and/or tumour debulking. Only then were the tumour capsules dissected away from the spinal cord. With the use of the operating microscope excellent access was afforded through a very limited exposure in which the spinal cord did not require to be manipulated to visualise the tumour. All patients made excellent recoveries without transient increase in existing deficits.

UNUSUAL, IRREMEDIAL CAUSES OF SPINAL CORD COMPRESSION: CASE REPORTS ON TWO YOUNG ADULTS

R Strachan, W Kelly, S Marks. Middlesbrough

Two rare cases of spinal cord compression were reported both occurring in young adult males as a result of nodular fasciitis and pedicular hypertrophy secondary to osteodystrophy respectively.

Firstly, a 42 year old man presented with acute cord compression over a two day period, two weeks after lifting a diesel engine. Bladder function was affected, and examination revealed a marked paraparesis. Myelography showed a complete block at T6 to T8. At surgery, the spinal cord was compressed posteriorly by soft, yellow, abnormal extradural tissue, extending both cranially and caudally beyond the laminectomy. Following decompression the patient regained full bladder control, and was now mobile with some support. Subsequent histology revealed nodular fasciitis involving the extradural tissues. Magnetic Resonance Imaging demonstrated a thinning of the spinal cord throughout its length, with involvement of the extradural tissues from cervical to lumbar region.

Secondly, an obese, slightly retarded 28 year old man with a long history of epilepsy presented with a mild progressive spastic paraparesis and urinary incontinence, two months after a fall. He was noted to have a low plasma $\mathrm{Ca}^{2+}$, with other endocrine abnormalities consistent with Albright's Hereditary Osteodystrophy with pseudohypoparathyroidism. Investigations revealed bony compression at the cervicodorsal and thoracolumbar junctions, and decompressive laminectomies at both these levels were carried out. Although his mobility improved, his lower limbs remained somewhat spastic, and he represented 18 months later with progressive spastic paraplegia despite maintenance of normal biochemistry. Investigations showed a marked overgrowth of the laminae and pedicles of the vertebral column, and an extensive dorso-lumbar laminectomy was carried out. He failed to improve, and CT myelography 
revealed persistent cord compression by the pedicles at the cervicodorsal junction and at D9 despite laminectomy. C6 to L5 laminectomy was completed-exploration of the cord at D9 revealed a myelomalacia. $\mathrm{He}$ is now confined to a wheelchair.

CRANIO-CERVICAL STABILISATION USING LUQUE/HARTSHILL RECTANGLES

AI Mackenzie, HT Marsh, BA Bell, D Uttley. Atkinson Morley's Hospital, London

Untreated cranio-cervical instability is associated with a high morbidity and a significant mortality. Existing methods using bone grafts, inter-laminar wires, or acrylic eventually produce stability, but require prolonged periods of immobility and have a high failure rate. The ideal method of fixation should provide for permanent correction of deformity and relief of symptoms, with immediate stabilisation, at a single procedure. The authors reported their use of posterior fixation of the occiput to a stable part of the cervical spine with a moulded metal rectangle (Luque/Hartshill rectangles) held in place by multiple sublaminar wires in 20 patients. Fourteen patients had pre-existing Atlanto-Axial instability and six had cord compression and would have become unstable after decompression. All operations were performed under general anaesthetic with awake intubation/positioning in 8 and 7 patients had a simultaneous decompression. Seventeen patients made an uncomplicated recovery and were mobilised three days postoperatively. Symptomatic and neurological improvement occurred in 70 per cent of all patients (average follow-up 15 months). Neurological complications occurred in four patients (20 per cent)-monoplegia (1), cerebellar infarction (2) and quadriplegia (1). Scrutiny of their presentations and operation failed to identify avoidable risk factors, except difficulties with sublaminar wiring. In all patients, stabilisation was achieved immediately, facilitating early mobilisation with a real chance of improvement.

UPPER CERVICAL STABILISATION: FRONT, BACK OR SIDES?

A Crockard, R Moskovich, J Ashraf. National Hospital for Nervous Diseases, London

Instability in the first three cervical vertebrae presents a difficult management problem. To leave may result in sudden death, to immobilise externally is associated with a high incidence of non-union and to fuse may be surgically exacting. The authors presented their experiences with various approaches for patients with single subluxation in otherwise healthy cervical spines. For many patients with widespread bony and ligamentous pathology, occipitocervical fixation (Ransford Loop) with and without fusion had been carried out. In five patients, a transoral bony fusion with a Dowel bone graft had been employed at $\mathrm{C} 1 / \mathrm{C} 2$ and $\mathrm{C} 2$ / C3. There had been no wound infection, and full bony union. Halo body immobilisation was not required. Posterior fixation had been employed with $\mathrm{Cl}, \mathrm{C} 2$ sublaminar wire fixation. One of the problems with sublaminar wiring techniques was the danger to the spinal cord during the passage of the wires. Sublaminar wire guides had been developed which ease the passage of the wires and protected the dura. The Halifax clamp system had been introduced recently which allowed firm fixation with minimal dural manipulation. Seven patients had been subjected to the technique with the addition of a bone-spacer on each side with very satisfactory results, good fixation, good union and no external fixation.

The Swiss technique of C1, C2 lateral mass screw fixation is the most neat anatomically of the methods but the most exacting surgically with close proximity of the vertebral artery, dura and $\mathrm{C} 2$ nerve roots. The method may have a place in the future neurosurgical armamentarium.

MALIGNANT SPINAL CORD COMPRESSION ASSOCIATED WITH VERTEBRAL COLLAPSE: A NEW TREATMENT METHOD

FG Johnston, HT Marsh, D Uttley. Atkinson Morley's Hospital, London

Thirty-four patients with metastatic tumours of the spinal column presenting with vertebral collapse and posterior element disease were treated by a single stage operation combining decompression of the vertebral body with posterior spinal instrumentation. The combination of plain radiography and CT scanning including two levels above and below the lesion proved most effective in planning the operative approach. Indications for surgery were neurological deficit in 32 and pain in two patients. Fourteen patients had pre-operative radiotherapy.

Effect of Operation on Ambulatory State:

\begin{tabular}{lll}
\hline & Preoperative & Postoperative \\
Ambulatory & 12 & 24 \\
Paraparetic & 20 & 7 \\
Paraplegic & 2 & 3 \\
\hline
\end{tabular}

Thirty three per cent of the ambulant patients postoperatively remained so for $\$ x$ months or more. Fifty per cent of ambula্xt patients remained so until death and thee patients remained ambulant after one to years. Twenty one percent of patients regained sphincter control and $65 \%$ had less pain. Surgical mortality was $15 \%$ (5 deat at 30 days; wound infection occurred in fôr and dehiscence in five, and $C 7$ radiculopaty after sublaminar wiring occurred in five patients. These results indicate that a singe stage postero-lateral decompression and stabilisation carried a good chance $\frac{5}{6}$

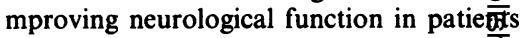
with malignant cord compression, with $\overline{\text { Thn }}$ operative morbidity and mortality compafable to that of laminectomy and representra dramatic improvement when compared with conventional methods of treatment.

ROUND TABLE DISCUSSION-SPINAL STABILISATION IN NEUROSURGERY A Crockard, RA Johnston, D Uttlēy, G Findlay

Discussion concentrated on the management of spinal metastases particularly 0 tte problems of the transthoracic approac $\overline{\text { the }}$ relative merits of metallic implants, bonf and acrylic grafts, the potential hazar $=0$ sublaminar wiring and new developments such as the Halifax screw. 